\title{
Miroslav Doupovec
}

Natural transformations between $T T T^{*} M$ and $T T^{*} T M$

Czechoslovak Mathematical Journal, Vol. 43 (1993), No. 4, 599-613

Persistent URL: http://dml.cz/dmlcz/128434

\section{Terms of use:}

(C) Institute of Mathematics AS CR, 1993

Institute of Mathematics of the Czech Academy of Sciences provides access to digitized documents strictly for personal use. Each copy of any part of this document must contain these Terms of use.

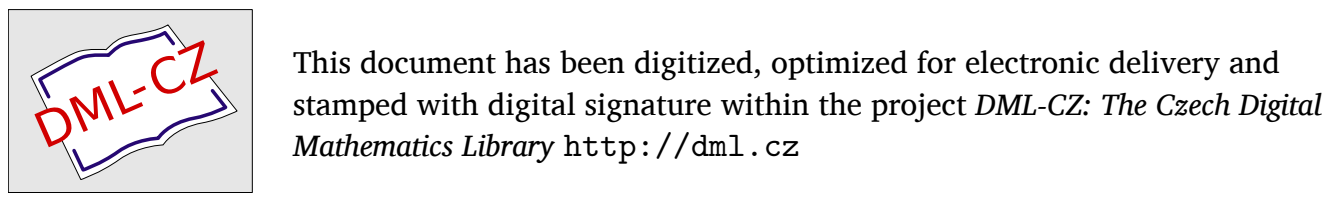




\title{
NATURAL TRANSFORMATIONS BETWEEN $T T T^{*} M$ AND $T T^{*} T M$
}

\author{
Miroslav Doupovec, Brno
}

(Received January 31, 1992)

\section{INTRODUCTION}

Starting from the canonical isomorphism $s: T T^{*} \rightarrow T^{*} T$ by Modugno and Stefani [10], Kolár and Radziszewski determined all natural transformations of $T T^{*}$ into $T^{*} T$, [7]. Analogously, all natural transformations between $T_{1}^{2} T^{*}$ and $T^{*} T_{1}^{2}$ were classified in [2] ( $T_{1}^{2}$ denotes the functor of 1-dimensional velocities of order 2).

In this paper we determine all natural transformations of $T T T^{*}$ into $T T^{*} T$ and interpret them geometrically. Further we show that all natural operators $T \rightsquigarrow T T T^{*}$ transforming vector fields on a manifold $M$ into vector fields on $T T^{*} M$ can be constructed from the flow operator by applying all natural transformations of $T T T^{*}$ into $T T T^{*}$ over the identity of $T T^{*}$. However this is the property of Weil functors, which is also satisfied for the non product-preserving functor $T T^{*}$. In the last section we describe all natural affinors on $T T^{*} M$.

All manifolds and maps are assumed to be smooth.

\section{Bundles of THE TyPE $H G F$}

Denote by $\mathscr{M} f$ the category of all manifolds and all smooth maps, by $\mathscr{M} f_{I}$ the category of all manifolds and their local diffeomorphisms, and by $\mathscr{M} f_{m}$ the category of $m$-dimensional manifolds and their local diffeomorphisms. We shall use the concept of a natural bundle on $\mathscr{M} f_{m}$ (or simply natural bundle) in the sense of Nijenhuis, [11]. If we replace the category $\mathscr{M} f_{m}$ by the category $\mathscr{M} f$, we obtain the

The author is indebted to Prof. I. Kolár for suggesting the problem and for many helpful comments 
concept of a bundle functor on $\mathscr{M} f$. Let $T$ or $T^{*}$ denote the tangent or cotangent bundle, respectively.

Consider a bundle functor $F$ on $\mathscr{M} f_{I}$ and two bundle functors $G, H$ on $\mathscr{M} f$. The bundle projection $p_{M}^{F}: F M \rightarrow M$ gives the induced mapping $H G p_{M}^{F}: H G F M \rightarrow$ $H G M$. Next, we have the bundle projections $p_{F M}^{G}: G F M \rightarrow F M, p_{G F M}^{H}: H G F M$ $\rightarrow G F M$ and the induced mapping $H p_{F M}^{G}: H G F M \rightarrow H F M$. Since $G$ and $H$ are bundle functors on $\mathscr{M} f$, the following diagram commutes:

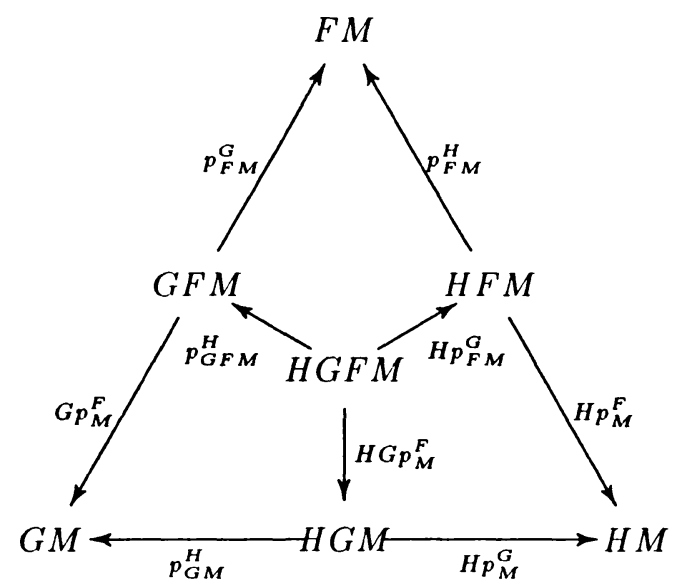

(Similar diagrams are used in [12] to describe some properties of the third tangent bundle TTTM .) In addition to the projections from $H G F M$ into $G F M, H G M$ and $H F M$ we have also the natural projections from $H G F M$ into $G M, H M$ and $F M$, which correspond to the diagonals of the commutative squares in (1). Further, let us define a set

$$
\begin{aligned}
(H, G, F) M= & \left\{(a, b, c) \in G F M \times H F M \times H G M ; p_{F M}^{G}(a)=p_{F M}^{H}(b)\right. \\
& \left.G p_{M}^{F}(a)=p_{G M}^{H}(c), H p_{M}^{F}(b)=H p_{M}^{G}(c)\right\} .
\end{aligned}
$$

One easily verifies that for every diffeomorphism $f: M \rightarrow N$ the map $H G F f$ is projectable over certain map $(H, G, F) f:(H, G, F) M \rightarrow(H, G, F) N$ satisfying $(H, G, F)(g \circ f)=(H, G, F) g \circ(H, G, F) f$. Moreover, if every $(H, G, F) M \rightarrow M$ is a fibered manifold, then $(H, G, F)$ is a bundle functor on $\mathscr{M} f_{I}$.

In the following example we describe $T T T^{*} M$ by means of a diagram of the type (1). We also introduce local coordinates on $T T T^{*} M$.

Example 1. While the tangent functor $T$ is defined on the whole category $\mathscr{M} f$, the cotangent functor $T^{*}$ is defined only on local diffeomorphisms. For every local diffeomorphism $f: M \rightarrow N$ we define $T^{*} f: T^{*} M \rightarrow T^{*} N$ in such a way that 
$T_{x}^{*} f: T_{x}^{*} M \rightarrow T_{f(x)}^{*} N$ is the inverse map of the dual map $\left(T_{x} f\right)^{*}: T_{f(x)}^{*} N \rightarrow T_{x}^{*} M$. Denote by $q_{M}: T^{*} M \rightarrow M, p_{M}: T M \rightarrow M$ the bundle projections. If $\pi: E \rightarrow M$ is a vector bundle, then there are two vector bundle structures on the tangent bundle $T E$, namely $p_{E}: T E \rightarrow E$ and $T \pi: T E \rightarrow T M$. So $p_{T \cdot M}, T q_{M}, p_{T T^{*} M}, T p_{T \cdot M}$ and $T T q_{M}$ are vector bundle projections. The canonical coordinates $x^{i}$ on $\mathbf{R}^{m}$ induce the additional coordinates $p_{i}$ on $T^{*} \mathbf{R}^{m}$ and $z^{i}=\mathrm{d} x^{i}, q_{i}=\mathrm{d} p_{i}$ on $T T^{*} \mathbf{R}^{m}$. Hence on $T T T^{*} \mathbf{R}^{m}$ we have local coordinates $x^{i}, p_{i}, z^{i}, q_{i}, X^{i}=\mathrm{d} x^{i}, P_{i}=\mathrm{d} p_{i}, Z^{i}=\mathrm{d} z^{i}$, $Q_{i}=\mathrm{d} q_{i}$. The space $T T T^{*} M$ can be characterized by means of the diagram

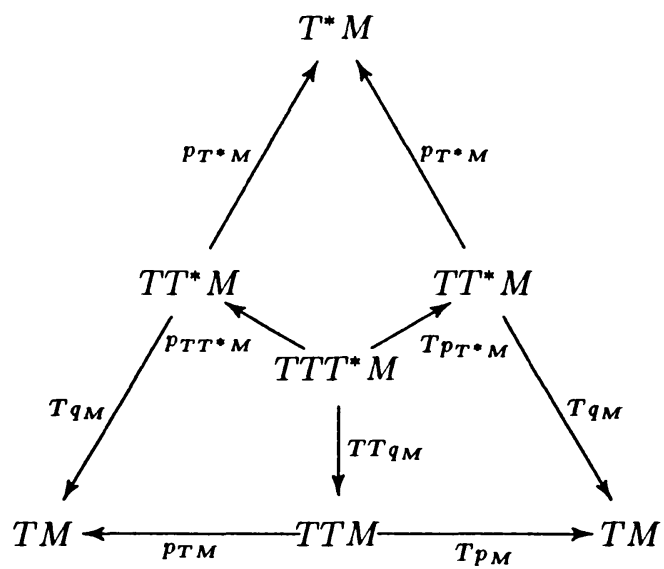

We have also defined the bundle $\left(T, T, T^{*}\right)$.

In the case of the diagram (1) in general it is not possible to interchange $F$ and $G$. But if there is a natural equivalence $h: F G \rightarrow G F$ of bundle functors defined in the category $\mathscr{M} f_{I}$ such that the diagram

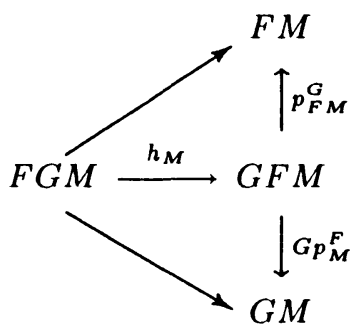

commutes then we can define an analogue of (1) also for the composition $H F G$. For example, in the case of $T T^{*} T$ we can take the natural equivalence $s: T T^{*} \rightarrow T^{*} T$ by Modugno and Stefani. 
Ex a mple 2. If $\pi: E \rightarrow M$ is a vector bundle, then on the cotangent bundle $q_{E}$ : $T^{*} E \rightarrow E$ there is another vector bundle structure $\varrho_{E^{*}}: T^{*} E \rightarrow E^{*}$ defined by the restriction of a linear map $T_{y} E \rightarrow \mathbf{R}$ to the vertical tangent space, which is identified with $E_{\pi(y)}$. In this way $q_{T M}$ and $\varrho_{T^{*} M}$ are vector bundle projections on $T^{*} T M$, so that $T T^{*} T M$ has three vector bundle structures $p_{T \cdot T M}, T q_{T M}$ and $T \varrho_{T}{ }^{*} M$. Let $w^{i}=\mathrm{d} x^{i}$ be the coordinates on $T \mathbf{R}^{m}$ induced by the canonical coordinates $x^{i}$ on $\mathbf{R}^{m}$. Then the expression $r_{i} \mathrm{~d} x^{i}+s_{i} \mathrm{~d} w^{i}$ determines the additional coordinates $r_{i}, s_{i}$ on $T^{*} T \mathbf{R}^{m}$. So $x^{i}, w^{i}, r_{i}, s_{i}, Y^{i}=\mathrm{d} x^{i}, W^{i}=\mathrm{d} w^{i}, R_{i}=\mathrm{d} r_{i}, S_{i}=\mathrm{d} s_{i}$ are local coordinates on $T T^{*} T \mathbf{R}^{m}$. Finally, we can describe the space $T T^{*} T M$ by means of

(3)

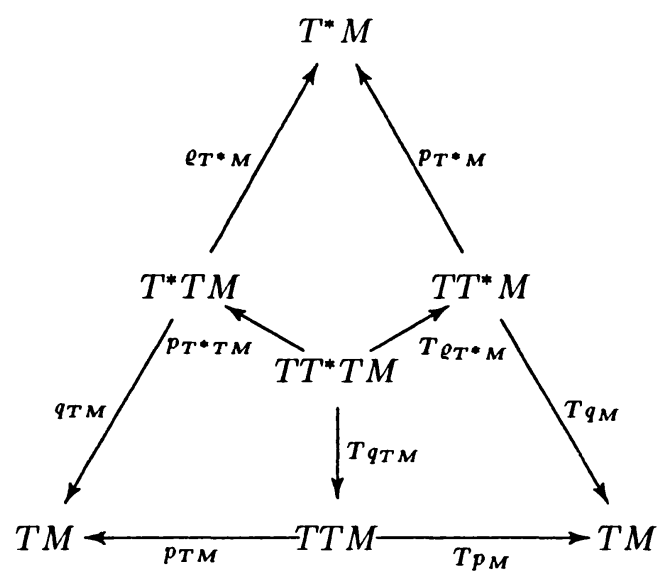

3. The analytical Result

In this section we determine all natural transformations $T T T^{*} \rightarrow T T^{*} T$. According to the general theory [9], we have to find all $G_{m}^{3}$-equivariant maps $\left(T T T^{*}\right)_{0} \rightarrow$ $\left(T T^{*} T\right)_{0}$ of the standard fibres, where $G_{m}^{r}$ means the group of all invertible $r$-jets on $\mathbf{R}^{m}$ with source and target zero. Denote by

$$
\left(a_{j}^{i}, a_{j k}^{i}, a_{j k l}^{i}\right)
$$

the canonical coordinates on $G_{m}^{3}$ and by a tilde the coordinates of the element inverse 602 
to (4) in $G_{m}^{3}$. Using standard evaluations we find the action of $G_{m}^{3}$ on $\left(T T T^{*}\right)_{0}$ :

$$
\begin{aligned}
\bar{p}_{i} & =\tilde{a}_{i}^{j} p_{j}, \quad \bar{q}_{i}=\tilde{a}_{i k}^{j} a_{l}^{k} z^{l} p_{j}+\tilde{a}_{i}^{j} q_{j}, \\
\bar{z}^{i} & =a_{j}^{i} z^{j}, \quad \bar{Z}^{i}=a_{j}^{i} Z^{j}-a_{l}^{i} \tilde{a}_{m n}^{l} a_{j}^{m} a_{k}^{n} X^{k} z^{j}, \\
\bar{X}^{i} & =a_{j}^{i} X^{j}, \quad \bar{P}_{i}=\tilde{a}_{i k}^{j} a_{l}^{k} X^{l} p_{j}+\tilde{a}_{i}^{j} P_{j}, \\
\bar{Q}_{i} & =\tilde{a}_{i k m}^{j} a_{n}^{m} a_{l}^{k} X^{n} z^{l} p_{j}-\tilde{a}_{i k}^{j} a_{l}^{k} \tilde{a}_{m n}^{l} a_{p}^{m} a_{q}^{n} X^{q} z^{p} p_{j}+ \\
& +\tilde{a}_{i k}^{j} a_{l}^{k} Z^{l} p_{j}+\tilde{a}_{i k}^{j} a_{l}^{k} z^{l} P_{j}+\tilde{a}_{i k}^{j} a_{l}^{k} X^{l} q_{j}+\tilde{a}_{i}^{j} Q_{j}
\end{aligned}
$$

The tangent map to the canonical isomorphism $s: T T^{*} \rightarrow T^{*} T$ by Modugno and Stefani is also an isomorphism. The equations of $s$ are

$$
w^{i}=z^{i}, r_{i}=q_{i}, s_{i}=p_{i}
$$

so that $T s: T T T^{*} \rightarrow T T^{*} T$ has the coordinate expression

$$
w^{i}=z^{i}, r_{i}=q_{i}, s_{i}=p_{i}, Y^{i}=X^{i}, S_{i}=P_{i}, W^{i}=Z^{i}, R_{i}=Q_{i} .
$$

Then the action of $G_{m}^{3}$ on $\left(T T^{*} T\right)_{0}$ can be derived from (5) and (7). Let us furhter denote

$$
I_{1}=p_{i} z^{i}, I_{2}=p_{i} X^{i}, I_{3}=p_{i} Z^{i}+P_{i} z^{i}, I_{4}=p_{i} Z^{i}+q_{i} X^{i} .
$$

One verifies directly that the expressions $I_{1}, I_{2}, I_{3}, I_{4}$, whose geometrical interpretation will be given later, are invariants with respect to the group $G_{m}^{2}$. By the following lemma, $I_{1}, I_{2}, I_{3}$ and $I_{4}$ even generate all $G_{m}^{2}$-invariants defined on the standard fibre of the bundle $\left(T, T, T^{*}\right)$.

Lemma 1. Let $m \geqslant 2$ and let $F$ be a smooth $G_{m}^{2}$-invariant function defined on the standard fibre of the bundle $\left(T, T, T^{*}\right)$. Then there is a smooth function $f$ : $\mathbf{R}^{4} \rightarrow \mathbf{R}$ such that

$$
F\left(z^{i}, X^{i}, p_{i}, q_{i}, P_{i}, Z^{i}\right)=f\left(I_{1}, I_{2}, I_{3}, I_{4}\right)
$$

Pro of. Suppose first $m=2$ and define $f$ by the formula

$$
f\left(y_{1}, y_{2}, y_{3}, y_{4}\right)=F\left(1,0 ; 0,1 ; y_{1}, y_{2} ; 0, y_{4} ; y_{3}, 0 ; 0,0\right) .
$$

There is a linear transformation transforming independent vectors $z$ and $X$ into $(1,0)$ and $(0,1)$, respectively. Further, (5) with $a_{j}^{i}=\delta_{j}^{i}$ gives

$$
\bar{q}_{i}=q_{i}+\tilde{a}_{i 1}^{j} p_{j}, \quad \bar{P}_{i}=P_{i}+\tilde{a}_{i 2}^{j} p_{j}, \quad \bar{Z}^{i}=Z^{i}-\tilde{a}_{12}^{i} .
$$


By the choice of $\tilde{a}_{12}^{i}$ we obtain $\bar{Z}^{i}=0$. Analogously, for $p_{1} \neq 0$ the choice of $\tilde{a}_{11}^{1}$ yields $\bar{q}_{1}=0$ and for $p_{2} \neq 0$ the choice of $\tilde{a}_{22}^{2}$ gives $\bar{P}_{2}=0$. Then $q_{2}=I_{4}$ and $P_{1}=I_{3}$. Hence (9) holds on open dense subset, so that it holds everywhere.

Now let $m>2$. By the tensor evaluation theorem from [9] there is a smooth function $f: \mathbf{R}^{9} \rightarrow \mathbf{R}$ such that

$$
F\left(z^{i}, X^{i}, p_{i}, q_{i}, P_{i}, Z^{i}\right)=f\left(p_{i} z^{i}, p_{i} X^{i}, p_{i} Z^{i}, q_{i} z^{i}, q_{i} X^{i}, q_{i} Z^{i}, P_{i} z^{i}, P_{i} X^{i}, P_{i} Z^{i}\right)
$$

Put

$$
F\left(z^{i}, X^{i}, p_{i}, q_{i}, P_{i}, Z^{i}\right)=f\left(I_{1}, I_{2}, I_{3}, I_{4}, q_{i} z^{i}, q_{i} Z^{i}, P_{i} X^{i}, P_{i} Z^{i}, p_{i} Z^{i}-q_{i} X^{i}-P_{i} z^{i}\right)
$$

We prove that the function $f$ depends only on the first four variables. The invariance on the kernel of the jet projection $G_{m}^{2} \rightarrow G_{m}^{1}$ reads

$$
\begin{aligned}
f\left(I_{1}, I_{2}, I_{3}, I_{4}, q_{i} z^{i},\right. & \left.q_{i} Z^{i}, P_{i} X^{i}, P_{i} Z^{i}, p_{i} Z^{i}-q_{i} X^{i}-P_{i} z^{i}\right) \\
& =f\left(I_{1}, I_{2}, I_{3}, I_{4}, \bar{q}_{i} z^{i}, \bar{q}_{i} \bar{Z}^{i}, \bar{P}_{i} X^{i}, \bar{P}_{i} \bar{Z}^{i}, p_{i} \bar{Z}^{i}-\bar{q}_{i} X^{i}-\bar{P}_{i} z^{i}\right) .
\end{aligned}
$$

Setting $z=(1,0, \ldots, 0), X=(0,1,0, \ldots, 0), Z=(0,0,1, \ldots, 0)$ we obtain

$$
\begin{aligned}
f\left(p_{1}, p_{2}, p_{3}+P_{1}, p_{3}+q_{2}, q_{1}, q_{3}, P_{2}, P_{3}, p_{3}-q_{2}-P_{1}\right) \\
=f\left(p_{1}, p_{2}, p_{3}+P_{1}, p_{3}+q_{2}, \tilde{a}_{11}^{j} p_{j}+q_{1},\left(\tilde{a}_{31}^{j} p_{j}+q_{3}\right)\left(-\tilde{a}_{21}^{3}+1\right), \tilde{a}_{22}^{j} p_{j}+P_{2},\right. \\
\left.\quad\left(\tilde{a}_{32}^{j} p_{j}+P_{3}\right)\left(-\tilde{a}_{21}^{3}+1\right), p_{3}\left(-\tilde{a}_{21}^{3}+1\right)-\left(\tilde{a}_{21}^{j} p_{j}+q_{2}\right)-\left(\tilde{a}_{12}^{j} p_{j}+P_{1}\right)\right) .
\end{aligned}
$$

If all $\tilde{a}_{j k}^{i}$ except $\tilde{a}_{11}^{1}$ are zero then

$$
\begin{aligned}
f\left(p_{1}, p_{2}, p_{3}+P_{1}, p_{3}+q_{2}, q_{1}, q_{3}, P_{2}, P_{3}, p_{3}-q_{2}-P_{1}\right) \\
\quad=f\left(p_{1}, p_{2}, p_{3}+P_{1}, p_{3}+q_{2}, q_{1}+\tilde{a}_{11}^{1} p_{1}, q_{3}, P_{2}, P_{3}, p_{3}-q_{2}-P_{1}\right) .
\end{aligned}
$$

This implies that the function $f$ is independent of the fifth variable. Analogously, by means of $\tilde{a}_{31}^{1}, \tilde{a}_{22}^{1}, \tilde{a}_{32}^{1}$ and $\tilde{a}_{12}^{2}$ we prove that $f$ does not depend on the sixth, seventh, eigth and nineth variable, respectively.

Now we shall look for the coordinate form of all $G_{m}^{3}$-equivariant maps $\left(T T T^{*} \mathbf{R}^{m}\right)_{0} \rightarrow\left(T T^{*} T \mathbf{R}^{m}\right)_{0}$. Consider first $w^{i}=w^{i}\left(p_{i}, z^{i}, q_{i}, X^{i}, P_{i}, Z^{i}, Q_{i}\right)$. Taking into account the equivariance with respect to the kernel of the jet projection $G_{m}^{3} \rightarrow G_{m}^{2}$ we obtain

$$
w^{i}\left(p_{i}, z^{i}, q_{i}, X^{i}, P_{i}, Z^{i}, Q_{i}\right)=w^{i}\left(p_{i}, z^{i}, q_{i}, X^{i}, P_{i}, Z^{i}, Q_{i}+\tilde{a}_{i k l}^{j} X^{l} z^{k} p_{j}\right)
$$


so that $w^{i}$ does not depend on $Q_{i}$. Quite analogously one can prove that $r_{i}, s_{i}, Y^{i}$, $S_{i}$ and $W^{i}$ are also independent of $Q_{i}$. Assume now $m>2$. The equivariance of $w^{i}$ with respect to the linear group $G_{m}^{1} \subset G_{m}^{3}$ implies that $w^{i}$ is a $G_{m}^{1}$-equivariant map $\mathbf{R}^{3 m} \times \mathbf{R}^{3 m *} \rightarrow \mathbf{R}^{m}$. By the tensor evaluation theorem [9] $w^{i}$ has the form

$$
\begin{aligned}
w^{i}= & \alpha\left(p_{i} z^{i}, p_{i} X^{i}, p_{i} Z^{i}, q_{i} z^{i}, q_{i} X^{i}, q_{i} Z^{i}, P_{i} z^{i}, P_{i} X^{i}, P_{i} Z^{i}\right) z^{i} \\
& +\beta\left(p_{i} z^{i}, \ldots, P_{i} Z^{i}\right) X^{i}+\gamma\left(p_{i} z^{i}, \ldots, P_{i} Z^{i}\right) Z^{i}
\end{aligned}
$$

where $\alpha, \beta, \gamma$ are smooth functions of nine variables. In the same way as in the proof of Lemma 1 we deduce that $\alpha=\alpha\left(I_{1}, I_{2}, I_{3}, I_{4}\right), \beta=\beta\left(I_{1}, I_{2}, I_{3}, I_{4}\right)$ and $\gamma=0$. Hence

$$
w^{i}=A\left(I_{1}, I_{2}, I_{3}, I_{4}\right) z^{i}+B\left(I_{1}, I_{2}, I_{3}, I_{4}\right) X^{i}
$$

with arbitrary smooth functions $A, B$ of four variables. For $m=2$ let us introduce an additional variable $\left(u_{1}, u_{2}\right) \in \mathbf{R}^{2 *}, \bar{u}_{i}=\tilde{a}_{i}^{j} u_{j}$. Then $w^{i}\left(p_{i}, z^{i}, q_{i}, X^{i}, P_{i}, Z^{i}\right) u_{i}$ is a $G_{2}^{2}$-invariant function and the expressions $I_{5}=u_{i} z^{i}, I_{6}=u_{i} X^{i}$ are $G_{2}^{2}$-invariants. Similarly to the proof of Lemma 1 one can deduce

Lemma 2. For every $G_{2}^{2}$-invariant smooth function $F\left(z^{i}, X^{i}, p_{i}, q_{i}, P_{i}, Z^{i}, u_{i}\right)$ there is a smooth function $f: \mathbf{R}^{6} \rightarrow \mathbf{R}$ such that

$$
F\left(z^{i}, X^{i}, p_{i}, q_{i}, P_{i}, Z^{i}, u_{i}\right)=f\left(I_{1}, I_{2}, I_{3}, I_{4}, I_{5}, I_{6}\right) .
$$

Thus, $w^{i} u_{i}=f\left(I_{1}, I_{2}, I_{3}, I_{4}, I_{5}, I_{6}\right)$. Differentiating this with respect to $u_{i}$ we get

$$
w^{i}=\frac{\partial f\left(I_{1}, \ldots, I_{6}\right)}{\partial x_{5}} z^{i}+\frac{\partial f\left(I_{1}, \ldots, I_{6}\right)}{\partial x_{6}} X^{i}
$$

where $x_{5}$ or $x_{6}$ denote the fifth or sixth component of $f$, respectively. Setting $u_{1}=0$, $u_{2}=0$ on the right hand side of the equation we obtain $w^{i}=\varphi\left(I_{1}, \ldots, I_{4}\right) z^{i}+$ $\psi\left(I_{1}, \ldots, I_{4}\right) X^{i}$, where

$$
\varphi\left(x_{1}, \ldots, x_{4}\right)=\left.\frac{\partial f\left(x_{1}, \ldots, x_{6}\right)}{\partial x_{5}}\right|_{x_{5}=x_{6}=0}, \psi\left(x_{1}, \ldots, x_{4}\right)=\left.\frac{\partial f\left(x_{1}, \ldots, x_{6}\right)}{\partial x_{6}}\right|_{x_{5}=x_{6}=0} .
$$

Therefore (10) holds also in the case $m=2$. Analogously,

$$
Y^{i}=C\left(I_{1}, \ldots, I_{4}\right) z^{i}+D\left(I_{1}, \ldots, I_{4}\right) X^{i}
$$


and also

$$
s_{i}=E\left(I_{1}, \ldots, I_{4}\right) p_{i}
$$

with arbitrary smooth functions $C, D, E: \mathbf{R}^{4} \rightarrow \mathbf{R}$.

Assume a map $r_{i}\left(p_{i}, z^{i}, q_{i}, X^{i}, P_{i}, Z^{i}\right)$ is in the form

$$
r_{i}=\alpha q_{i}+\beta P_{i}+\tilde{r}_{i}\left(p_{i}, z^{i}, q_{i}, X^{i}, P_{i}, Z^{i}\right)
$$

Using equivariance on the kernel of the jet projection $G_{m}^{2} \rightarrow G_{m}^{1}$ we obtain $\alpha=A E$, $\beta=B E$. Then the full equivariance reads $\tilde{a}_{i}^{j} \tilde{r}_{j}\left(p_{i}, \ldots, Z^{i}\right)=\tilde{r}_{i}\left(\bar{p}_{i}, \ldots, \bar{Z}^{i}\right)$, so that $\tilde{r}_{i}$ has the same transformation law as $s_{i}$. Hence $\tilde{r}_{i}=F\left(I_{1}, \ldots, I_{4}\right) p_{i}$ and

$$
r_{i}=A E q_{i}+B E P_{i}+F p_{i}
$$

where $F$ is another smooth function of four variables. Quite analogously,

$$
S_{i}=C E q_{i}+D E P_{i}+G p_{i}
$$

where $G=G\left(I_{1}, \ldots, I_{4}\right)$ is a smooth function. Applying a procedure similar to that used in the case of $r_{i}$ we prove

$$
W^{i}=(A D+B C) Z^{i}+H z^{i}+K X^{i}
$$

$$
R_{i}=E(A D+B C) Q_{i}+(A G+E H+C F) q_{i}+(B G+E K+D F) P_{i}+L p_{i}
$$

and the additional conditions $A C=0, B D=0$. Thus, we have deduced

Proposition 1. Let $m \geqslant 2$. Then all natural transformations $T T T^{*} \rightarrow T T^{*} T$ are of the form

$$
\begin{aligned}
w^{i} & =A z^{i}+B X^{i} \\
Y^{i} & =C z^{i}+D X^{i} \\
s_{i} & =E p_{i} \\
r_{i} & =A E q_{i}+B E P_{i}+F p_{i}, \\
S_{i} & =C E q_{i}+D E P_{i}+G p_{i}, \\
W^{i} & =(A D+B C) Z^{i}+H z^{i}+K X^{i}, \\
R_{i} & =E(A D+B C) Q_{i}+(A G+E H+C F) q_{i}+(B G+E K+D F) P_{i}+L p_{i}
\end{aligned}
$$


where $A, B, C, D, E, F, G, H, K, L$ are arbitrary smooth functions of four variables viewed as functions of the invariants $I_{1}, I_{2}, I_{3}, I_{4}$. Moreover, the functions $A, B$, $C, D$ satisfy the conditions

$$
A C=0, \quad B D=0 .
$$

\section{Geometrical interpretation}

We first describe a simple geometric construction of the invariants $I_{1}, I_{2}, I_{3}, I_{4}$ given by (8). Every $A \in T T T^{*} M$ is a vector tangent to a curve $\gamma(t): \mathbf{R} \rightarrow T T^{*} M$ at $t=0$. Then $\alpha(t):=p_{T^{*} M} \circ \gamma(t): \mathbf{R} \rightarrow T^{*} M$ and $\beta(t):=T q \circ \gamma(t): \mathbf{R} \rightarrow T M$, so that we can evaluate $\langle\alpha(t), \beta(t)\rangle$. The invariants (8) can be constructed as follows:

$$
\begin{aligned}
& I_{1}=\left.\langle\alpha(t), \beta(t)\rangle\right|_{t=0}=\left\langle p_{T^{*} M} \circ p_{T T^{*} M}(A), T q \circ p_{T T^{*} M}(A)\right\rangle, \\
& I_{2}=\left\langle p_{T^{*} M} \circ p_{T T^{*} M}(A), T q \circ T p_{T^{*} M}(A)\right\rangle, \\
& I_{3}=\left.\frac{\mathrm{d}}{\mathrm{d} t}\right|_{0}\langle\alpha(t), \beta(t)\rangle, \quad I_{4}=I_{3} \circ i_{T^{*} M}
\end{aligned}
$$

where $i_{T^{*} M}: T T T^{*} M \rightarrow T T T^{*} M$ is the canonical involution.

Now we present a geometric interpretation of Proposition 1. Let us notice that the isomorphism $T s_{M}: T T T^{*} M \rightarrow T T^{*} T M$ given by (7) corresponds to the constant values $A=D=E=1, B=C=F=G=H=K=L=0$ in (17). We have the following injection $\tilde{j}: T^{*} M \rightarrow T^{*} T M$. Take any vector $B \in T_{O_{x}} T M$, where $O_{x}$ is a zero vector over $x \in M$. Then $B^{\prime}=T p_{M}(B) \in T_{x} M$, so that we can define $\tilde{j}(A)(B):=\left\langle A, B^{\prime}\right\rangle$ for arbitrary $A \in T^{*} M$. Next, the composition $s_{M}^{-1} \circ \tilde{j}$ gives an injection $j_{1}: T^{*} M \rightarrow T T^{*} M,\left(x^{i}, p_{i}\right) \mapsto\left(x^{i}, 0,0, p_{i}\right)$. Moreover, denote by $j_{2}$ : $T T^{*} M \rightarrow T T T^{*} M$ the kernel injection, $\left(x^{i}, p_{i}, z^{i}, q_{i}\right) \mapsto\left(x^{i}, p_{i}, 0,0,0,0, z^{i}, q_{i}\right)$.

Starting from an arbitrary element

$$
\left(x^{i}, p_{i}, z^{i}, q_{i}, X^{i}, P_{i}, Z^{i}, Q_{i}\right) \in T T T^{*} M
$$

we construct the corresponding element (17) in $T T^{*} T M$. We proceed in three steps.

1. First we recall the equations of all natural transformations $t_{M}:$ TTTM $\rightarrow$ TTTM, see e.g. [5]. Let $x^{i}, a^{i}=\mathrm{d} x^{i}, b^{i}=\mathrm{d} x^{i}, c^{i}=\mathrm{d} a^{i}$ be the canonical coordinates on $T T \mathbf{R}^{m}$. Then the coordinate form of $t_{M}$ is

$$
\begin{aligned}
& \bar{a}^{i}=A a^{i}+B b^{i}, \\
& \bar{b}^{i}=C a^{i}+D b^{i}, \\
& \bar{c}^{i}=H a^{i}+K b^{i}+(A D+B C) c^{i}
\end{aligned}
$$


where $A, B, C, D, H, K$ are arbitrary real numbers satisfying $A C=0, B D=0$. Transforming (19) into $T T T^{*} M$ by means of $t_{T * M}: T T T^{*} M \rightarrow T T T^{*} M$ we have

$$
\begin{aligned}
& \left(x^{i}, p_{i}, A z^{i}+B X^{i}, A q_{i}+B P_{i}, C z^{i}+D X^{i}, C q_{i}+D P_{i}\right. \\
& \left.\quad(A D+B C) Z^{i}+H z^{i}+K X^{i},(A D+B C) Q_{i}+H q_{i}+K P_{i}\right) \in T T T^{*} M
\end{aligned}
$$

Furthermore, multiplying this by $E$ on the vector bundle $T T q: T T T^{*} M \rightarrow T T M$ we obtain

$$
\begin{aligned}
& \left(x^{i}, E p_{i}, A z^{i}+B X^{i}, A E q_{i}+B E P_{i}, C z^{i}+D X^{i}, C E q_{i}+D E P_{i}\right. \\
& \left.(A D+B C) Z^{i}+H z^{i}+K X^{i}, E(A D+B C) Q_{i}+H E q_{i}+K E P_{i}\right)
\end{aligned}
$$

2. Now we construct the term with $L$. Multiplying $\left(x^{i}, p_{i}\right) \in T^{*} M$ by $L$ on the vector bundle $T^{*} M \rightarrow M$ we have $\left(x^{i}, L p_{i}\right) \in T^{*} M$. Further, applying the injection $j_{1}: T^{*} M \rightarrow T T^{*} M$ we get

$$
\left(x^{i}, 0,0, L p_{i}\right) \in T T^{*} M .
$$

On the other hand, the injection of $\left(x^{i}, E p_{i}\right) \in T^{*} M$ into $T T^{*} M$ by means of the zero section gives

$$
\left(x^{i}, E p_{i}, 0,0\right) \in T T^{*} M .
$$

Evaluating the sum of (22) and (23) with respect to the vector bundle structure $T T^{*} M \rightarrow T M$ we obtain $\left(x^{i}, E p_{i}, 0, L p_{i}\right)$. Next, the kernel injection $j_{2}$ yields

$$
\left(x^{i}, E p_{i}, 0,0,0,0,0, L p_{i}\right) \in T T T^{*} M .
$$

Moreover, using the bundle projection $p_{T T * M}$ applied on (21) and then the zero section $T T^{*} M \rightarrow T T T^{*} M$ we obtain

$$
\left(x^{i}, E p_{i}, A z^{i}+B x^{i}, A E q_{i}+B E P_{i}, 0,0,0,0\right) \in T T T^{*} M .
$$

The sum of (24) and (25) on the vector bundle $T p_{T * M}$ is

$$
\left(x^{i}, E p_{i}, A z^{i}+B X^{i}, A E q_{i}+B E P_{i}, 0,0,0, L p_{i}\right) .
$$

Finally, we add this to (21) on the vector bundle structure $p_{T T^{*} M}$,

$$
\begin{aligned}
& \left(x^{i}, E p_{i}, A z^{i}+B X^{i}, A E q_{i}+B E P_{i}, C z^{i}+D X^{i}, C E q_{i}+D E P_{i}\right. \\
& \left.\quad(A D+B C) Z^{i}+H z^{i}+K X^{i}, E(A D+B C) Q_{i}+H E q_{i}+K E P_{i}+L p_{i}\right) .
\end{aligned}
$$


3. It remains to discuss the terms with $F$ and $G$. Denote by $s_{T M}: T T^{*} T M \rightarrow$ $T^{*} T T M$ the isomorphism $s$ by Modugno and Stefani, which is applied to $T M$. Then the composition $\left(T s_{M}\right)^{-1} \circ\left(s_{T M}\right)^{-1}: T^{*} T T M \rightarrow T T T^{*} M$ is also an isomorphism, which we will denote by $l_{M}$. If $a^{i}=\mathrm{d} x^{i}, b^{i}=\mathrm{d} x^{i}, c^{i}=\mathrm{d} a^{i}$ are the induced coordinates on $T T \mathbf{R}^{m}$, then the expressions $\alpha_{i} \mathrm{~d} x^{i}+\beta_{i} \mathrm{~d} a^{i}+\gamma_{i} \mathrm{~d} b^{i}+\delta_{i} \mathrm{~d} c^{i}$ determine the additional coordinates $\alpha_{i}, \beta_{i}, \gamma_{i}, \delta_{i}$ on $T^{*} T T \mathbf{R}^{m}$. We have the following inclusion $k_{M}: T T M \times_{T M} T^{*} T M \rightarrow T^{*} T T M$,

$$
\begin{aligned}
\left(x^{i}, z^{i}, X^{i}, Z^{i}, r_{i} \mathrm{~d} x^{i}+s_{i} \mathrm{~d} z^{i}\right) & \\
& \mapsto\left(a^{i}=z^{i}, b^{i}=X^{i}, c^{i}=Z^{i}, \alpha_{i}=r_{i}, \beta_{i}=s_{i}, \gamma_{i}=\delta_{i}=0\right) .
\end{aligned}
$$

The $T T q$-projection of (26) is

$$
\left(x^{i}, A z^{i}+B X^{i}, C z^{i}+D X^{i},(A D+B C) Z^{i}+H z^{i}+K X^{i}\right) \in T T M
$$

and the $p_{T T} \cdot M$-projection of $(20)$ is

$$
\left(x^{i}, p_{i}, A z^{i}+B X^{i}, A q_{i}+B p_{i}\right) \in T T^{*} M .
$$

Multiplying by $G$ on the vector bundle structure $T T^{*} M \rightarrow T M$ we get

$$
\left(x^{i}, G p_{i}, A z^{i}+B X^{i}, A G q_{i}+B G p_{i}\right) \in T T^{* *} M,
$$

which we map into $T^{*} T M$ by means of $s_{M}$. Hence we can apply inclusion $k_{M}$ to this element and to (27). Finally, transforming the result into $T T T^{*} M$ by means of $l_{M}$ we obtain

$$
\begin{aligned}
\left(x^{i}, 0, A z^{i}+B X^{i}, 0, C z^{i}+D X^{i}, G p_{i}\right. \\
\left.(A D+B C) Z^{i}+H z^{i}+K X^{i}, A G q_{i}+B G P_{i}\right) .
\end{aligned}
$$

Quite analogously, using the canonical involution $i_{T} \cdot M$ we have

$$
\begin{aligned}
\left(x^{i}, 0, A z^{i}+B X^{i}, F p_{i}, C z^{i}+\right. & D X^{i}, 0 \\
& \left.(A D+B C) Z^{i}+H z^{i}+K X^{i}, C F q_{i}+D F P_{i}\right) .
\end{aligned}
$$

Then the sum of $(28),\left(28^{\prime}\right)$ and (26) with respect to the vector bundle structure $T T q: T T T^{*} M \rightarrow T T M$ is

$$
\begin{gathered}
\left(x^{i}, E p_{i}, A z^{i}+B X^{i}, A E q_{i}+B E P_{i}+F p_{i}, C z^{i}+D X^{i}\right. \\
C E q_{i}+D E P_{i}+G p_{i},(A D+B C) Z^{i}+H z^{i}+K X^{i} \\
\left.E(A D+B C) Q_{i}+(H E+A G+C F) q_{i}+(K E+B G+D F) P_{i}+L p_{i}\right) .
\end{gathered}
$$


Transforming this into $T T^{*} T M$ by means of the isomorphism $T s_{M}$ we obtain (17) with constants $A, \ldots, L$. Finally, replacing the numbers $A, \ldots, L$ by arbitrary smooth functions of the invariants $I_{1}, I_{2}, I_{3}, I_{4}$ we complete the geometrical construction of (17).

$\mathrm{R} \mathrm{e} \mathrm{m} \mathrm{a} \mathrm{r} \mathrm{k.} \mathrm{In} \mathrm{addition} \mathrm{to} \mathrm{the} \mathrm{natural} \mathrm{equivalence} s: T T^{*} \rightarrow T^{*} T$ we also have a classical natural equivalence between $T T^{*}$ and $T^{*} T^{*}$, see e.g. [7]. So we can construct natural equivalences on all arrows of the diagram

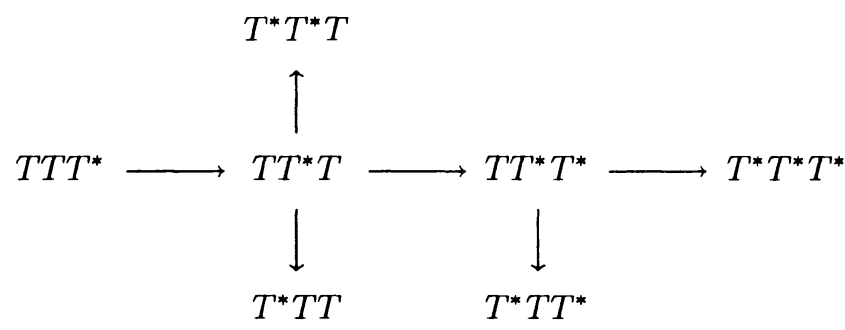

Hence the problem of finding all natural transformations between any two of the functors $T T T^{*}, T T^{*} T, T T^{*} T^{*}, T^{*} T^{*} T^{*}, T^{*} T^{*} T, T^{*} T T, T^{*} T T^{*}$ reduces to Proposition 1. In particular, all natural transformations of this type depend on eight arbitrary smooth functions of four variables. This is not true for the functor TTT, which is defined on the whole category $\mathscr{M} f$ and preserves products. According to the general theory [3], every product-preserving functor on $\mathscr{M} f$ is a Weil functor determined by a local algebra in the sense of $\mathrm{A}$. Weil, and the natural transformations between two such functors are in bijection with the homomorphisms of the local algebras in question. Hence the natural transformations of TTT into itself depend on a finite number of real parameters.

\section{NATURAL OPERATORS $T \rightsquigarrow T T T^{*}$}

Let $F$ be a natural bundle. Denote by $\mathscr{F}: T \rightsquigarrow T F$ the flow operator transforming every vector field $X$ on $M$ into its flow prolongation $\mathscr{F} X=\left.\frac{\mathrm{d}}{\mathrm{d} t}\right|_{0}(F(\exp t X))$, where $\exp t X$ means the flow of $X$. If $F$ is a Weil functor, then all natural operators $T \leadsto T F$ can be constructed from the flow operator $\mathscr{F}$ by applying all natural transformations $T F \rightarrow T F$ over the identity of $F$, [6]. But this does not necessarily hold for non product-preserving functors in general, the functor $T^{2}$ of the second order tangent vectors being the simplest counterexample, [1]. The functor $T T^{*}$ is another example of a non product-preserving functor. We prove 
Proposition 2. Let $m \geqslant 2$. Then all natural operators $T \leadsto T T T^{*}$ are of the form $U \circ \mathscr{T} \mathscr{T}^{*}$, where $\mathscr{T ~ T}^{*}: T \rightsquigarrow T T T^{*}$ is the flow operator and $U: T T T^{*} \rightarrow T T T^{*}$ is an arbitrary natural transformation over the identity of $T T^{*}$.

Proof. If $J: T T T^{*} \rightarrow T T^{*} T$ are all natural transformations from Proposition 1 , then the composition $(T s)^{-1} \circ J$ expresses all natural transformations $T T T^{*} \rightarrow$ $T T T^{*}$. Putting $A=E=1, B=F=C=0$ in (17) we have the following equations of all natural transformations $U: T T T^{*} \rightarrow T T T^{*}$ over the identity of $T T^{*}$ :

$$
\begin{aligned}
& \bar{X}^{i}=D X^{i}, \\
& \bar{P}_{i}=D P_{i}+G p_{i}, \\
& \bar{Z}^{i}=D Z^{i}+H z^{i}+K X^{i}, \\
& \bar{Q}_{i}=D Q_{i}+G q_{i}+H q_{i}+K P_{i}+L p_{i} .
\end{aligned}
$$

On the other hand, Kobak determined all natural operators $T \leadsto T T T^{*}$, provided $m \geqslant 2$, [4]. It is easy to verify that the composition $U \circ \mathscr{T}^{*}$ coincides with Kobak's result.

\section{Natural afFinors on $T T^{*} M$}

We first recall the concept of a natural affinor on a natural bundle, [8]. In general, an affinor $Q$ on a manifold $M$ means a tensor field of type $(1,1)$, i.e. a linear morphism $Q: T M \rightarrow T M$ over the identity of $M$. A natural affinor on a natural bundle $F$ is a system of affinors $Q_{M}: T F M \rightarrow T F M$ for every $m$-manifold $M$ satisfying

$$
T F f \circ Q_{M}=Q_{N} \circ T F f
$$

for every local diffeomorphism $f: M \rightarrow N$.

By [8] natural affinors can be used in the theory of torsions of generalized connections on natural bundles. In this context it is useful to determine all natural affinors on some natural bundles. For example, if $F$ is a Weil bundle, then all natural affinors on $F M$ are parametrized by $F \mathbf{R}$. In particular, all natural affinors on $T M$ form a 2-parameter family linearly generated by the identity affinor $I_{T M}$ and by $\alpha_{T M}$ : $T T M \rightarrow T T M,\left(x^{i}, y^{i}, \mathrm{~d} x^{i}, \mathrm{~d} y^{i}\right) \mapsto\left(x^{i}, y^{i}, 0, \mathrm{~d} x^{i}\right)$. In [8] the authors also determined all natural affinors on $T^{*} M$, which is not a Weil bundle.

The aim of this section is to classify all natural affinors on $T T^{*} M$. Two simplest examples are the identity affinor $I_{T T \cdot M}$ and $\alpha_{T T \cdot M}$. Next, we have the following 
injections $T T^{*} M \rightarrow T\left(T T^{*} M\right)$ :

$$
\begin{aligned}
& \beta:\left(x^{i}, p_{i}, z^{i}, q_{i}\right) \mapsto\left(x^{i}, p_{i}, z^{i}, q_{i}, 0,0, z^{i}, q_{i}\right), \\
& \gamma:\left(x^{i}, p_{i}, z^{i}, q_{i}\right) \mapsto\left(x^{i}, p_{i}, z^{i}, q_{i}, 0,0,0, p_{i}\right), \\
& \delta:\left(x^{i}, p_{i}, z^{i}, q_{i}\right) \mapsto\left(x^{i}, p_{i}, z^{i}, q_{i}, 0, p_{i}, 0, q_{i}\right) .
\end{aligned}
$$

Obviously, $\beta$ is the well-known injection $T Q \rightarrow T T Q,\left(u^{\alpha}, v^{\alpha}\right) \mapsto\left(u^{\alpha}, v^{\alpha}, 0, v^{\alpha}\right)$, where we identify the vertical tangent bundle $V T Q$ with $T Q \oplus T Q$. In other words, section $\beta$ is the Liouville vector field of the bundle $T T^{*} M \rightarrow T^{*} M$. Quite analogously, $\delta$ corresponds to the Liouville vector field of the bundle $T^{*} T M \rightarrow T M$, provided we identify $T T^{*} M$ with $T^{*} T M$ by means of $s_{M}$. Finally, $V T^{*} M=T^{*} M \oplus T^{*} M$ so that we have a subbundle $T T^{*} M \oplus T^{*} M \oplus T^{*} M$ in $V\left(T T^{*} M\right)=T T^{*} M \oplus T T^{*} M$. In this way $\gamma$ maps $B \in T T^{*} M$ into $\left(B, 0, p_{T^{*} M}(B)\right) \in T T T^{*} M$.

Multiplying $\beta, \gamma$ and $\delta$ by the invariants $I_{2}, I_{3}, I_{4}$ we obtain the natural affinors

$$
\begin{array}{lll}
\beta_{1}=I_{2} \beta, & \beta_{2}=I_{3} \beta, & \beta_{3}=I_{4} \beta, \\
\gamma_{1}=I_{2} \gamma, & \gamma_{2}=I_{3} \gamma, & \gamma_{3}=I_{4} \gamma, \\
\delta_{1}=I_{2} \delta, & \delta_{2}=I_{3} \delta, & \delta_{3}=I_{4} \delta .
\end{array}
$$

We will prove that these affinors together with $I_{T T * M}$ and $\alpha_{T T}{ }_{M}$ generate all natural affinors on $T T^{*} M$.

Proposition 3. Let $m \geqslant 2$. Then all natural affinors on $T T^{*} M$ are of the form

$$
\begin{aligned}
f_{1} I_{T T \cdot M}+f_{2} \alpha_{T T \cdot M}+f_{3} \beta_{1}+ & f_{4} \beta_{2}+f_{5} \beta_{3} \\
& +f_{6} \gamma_{1}+f_{7} \gamma_{2}+f_{8} \gamma_{3}+f_{9} \delta_{1}+f_{10} \delta_{2}+f_{11} \delta_{3}
\end{aligned}
$$

where $f_{i}=f_{i}\left(I_{1}\right)$ are arbitrary smooth functions of one variable viewed as functions of the invariant $I_{1}, i=1, \ldots, 11$.

Proof. All natural transformations $U: T T T^{*} \rightarrow T T T^{*}$ over the identity of $T T^{*}$ are of the form (29). The linearity condition for $U$ implies that the functions $D$ and $K$ depend only on $I_{1}$ and that the functions $G, H$ and $L$ are linear in $X^{i}, Z^{i}$, $P_{i}$. Hence $G, H, L$ must be linear in the last three variables $I_{2}, I_{3}, I_{4}$. Therefore the equations of all natural affinors on $T T^{*} M$ are

$$
\begin{aligned}
\bar{X}^{i}= & f_{1} X^{i} \\
\bar{P}_{i}= & f_{1} P_{i}+\left(f_{9} I_{2}+f_{10} I_{3}+f_{11} I_{4}\right) p_{i} \\
\bar{Z}^{i}= & f_{1} Z^{i}+\left(f_{3} I_{2}+f_{4} I_{3}+f_{5} I_{4}\right) z^{i}+f_{2} X^{i} \\
\bar{Q}_{i}= & f_{1} Q_{i}+\left(f_{9} I_{2}+f_{10} I_{3}+f_{11} I_{4}\right) q_{i} \\
& +\left(f_{3} I_{2}+f_{4} I_{3}+f_{5} I_{4}\right) q_{i}+f_{2} P_{i}+\left(f_{6} I_{2}+f_{7} I_{3}+f_{8} I_{4}\right) p_{i} .
\end{aligned}
$$


However, this is the coordinate form of (30).

\section{References}

[1] M. Doupovec: Natural operators transforming vector fields to the second order tangent bundle, Čas. pěst. mat. 115 (1990), 64-72.

[2] $M$. Doupovec: Natural transformations between $T_{1}^{2} T^{*} M$ and $T^{*} T_{1}^{2} M$, Ann. Polon. Math. 56 (1991), 67-77.

[3] G. Kainz, P.W. Michor: Natural transformations in differential geometry, Czech. Math. J. 37 (1987), 584-607.

[4] P. Kobak: Natural liftings of vector fields to tangent bundles of bundles of 1-forms, Mathematica Bohemica 116 (1991), 319-326.

[5] I. Kolár: Natural transformations of the second tangent functor into itself, Arch. Math.(Brno) $X X$ (1984), 169-172.

[6] I. Kolár: On the natural operators on vector fields, Ann. Global Anal. Geom. 6 (1988), 109-117.

[7] I. Kolár, Z. Radziszewski: Natural transformations of second tangent and cotangent functors, Czech. Math. J. 38(113) (1988), 274-279.

[8] I. Kolár, M. Modugno: Torsions of connections on some natural bundles, Differential Geometry and its Applications 2 (1992), 1-16.

[9] I. Kolár, P.W. Michor, J. Slovák: Natural operations in differential geometry, Springer Verlag, 1993.

[10] M. Modugno, G. Stefani: Some results on second tangent and cotangent spaces, Quaderni dell' Instituto di Matematica dell' Universita di Lecce Q.16 (1978).

[11] A. Nijenhuis: Natural bundles and their general properties, Diff. Geometry in honor of K. Yano, Kinokuniya,Tokyo (1972), 317-334.

[12] J. E. White: The method of iterated tangents with applications in local Riemannian geometry, Pitman Press, London, 1982.

Author's address: Department of Mathematics, Technical University of Brno, Technická 2, 61669 Brno, Czech Republic. 